\title{
Gain-Scheduled Drive-based Damping Control for Industrial Robots
}

This paper was downloaded from TechRxiv (https://www.techrxiv.org).

LICENSE

CC BY-SA 4.0

SUBMISSION DATE / POSTED DATE

23-12-2021 / 28-12-2021

\section{CITATION}

Mesmer, Patrick; Hinze, Christoph; Lechler, Armin; Verl, Alexander (2021): Gain-Scheduled Drive-based Damping Control for Industrial Robots. TechRxiv. Preprint. https://doi.org/10.36227/techrxiv.17429429.v1

DOI

10.36227/techrxiv.17429429.v1 


\title{
Gain-Scheduled Drive-based Damping Control for Industrial Robots
}

\author{
Patrick Mesmer, Christoph Hinze, Armin Lechler, and Alexander Verl, Fellow, IEEE
}

\begin{abstract}
The drivetrain flexibility of industrial robots limits their accuracy. To open up new areas of application for industrial robots, an increased dynamic path accuracy has to be obtained. Therefore, this paper addresses this issue by a gain-scheduled drive-based damping control for industrial robots with secondary encoders. For this purpose, a linear parameter-varying (LPV) model is derived as well as a system identification method is presented. Based on this, a gain-scheduled drive-based LPV damping control design is proposed, which guarantees stability and performance under variation of the manipulator configuration. The control performance of the approach is experimentally validated for the three base joints of a KUKA KR210-2 industrial robot. The approach realizes a trade-off between ease of implementation and control performance as well as robustness.
\end{abstract}

Index Terms-Motion control, flexible joint robots, linear parameter varying, structured $\mathrm{H}$-infinity synthesis.

\section{INTRODUCTION}

$\mathbf{S}$ INCE the 1980s, it is well known that the drivetrain flexibility of industrial robots (IRs) limits the achievable dynamic path accuracy [1] and therefore their area of application. To open up new application fields, such as milling [2], [3], cutting [4] or additive manufacturing [5], an increased dynamic path accuracy has to be obtained.

The issue of improved control performance of IRs has been continuously addressed by researchers over the past decades [6]-[10]. Nowadays, this topic is still being studied due to increasing accuracy requirements. For applications without process forces, pre-shaping of the input trajectories avoids excitation of flexible modes [11]. However, adjusted input trajectories do not affect the disturbance rejection behavior.

Therefore, various advanced feedback controllers under a rigid body assumption have been proposed, such as model predictive control [12], active disturbance rejection control [13], neural adaptive PID $^{2}$ control [14] and PD control with an adaptive rigid body dynamics compensation [15]. However, the potential of these approaches is limited by the fact that they do not explicitly account for the joint elasticity in the control design. Hence, controllers considering the joint elasticity have been proposed, e.g. backstepping control [16], Lyapunov-based adaptive neural network control [17] as well as two-time scale

Manuscript received Month xx, 2xxx; revised Month xx, xxxx; accepted Month x, xxxx. This work was supported by the German Research Foundation within the project $\mathrm{N}^{\mathrm{o}}: 290804444$.

Patrick Mesmer, Christoph Hinze, Armin Lechler and Alexander Verl are with the Institute for Control Engineering of Machine Tools and Manufacturing Units, University of Stuttgart, Stuttgart 70174, Germany (e-mail: \{patrick.mesmer; christoph.hinze; armin.lechler; alexander.verl\} @isw.unistuttgart.de) control using singular perturbation theory [18]. In addition, the activities concerning the DLR's lightweight robots [19] are noteworthy, especially their model-based feedback controllers with motor-side position and joint-side torque measurement [20] as well as with joint-side torque and position measurement [21].

But to date, this topic has not been fully addressed for IRs with heavy payloads. In such IRs, cycloidal drives are usually installed instead of harmonic drives as precision transmission [22]. As a consequence, these kinds of IRs exhibit uncertain drivetrain dynamics, which necessitate robust control approaches [23]. Hence, P-PI cascade control with motor-side position and velocity feedback [10] is still commonly used in the industry [24].

Recently, so-called secondary encoders (SEs) for joint-side position measurement gained popularity in the industry. The robot manufacturer Fanuc utilizes SEs in an additional control loop to compensate the joint torsion at low velocities to increase the static positioning accuracy [25]. The use of SEs for the position control of a P-PI cascade control increases the absolute accuracy and improves the low frequency disturbance rejection behavior [26].

For many practical, nonlinear engineering applications, gain scheduling has proven to be a successful control method [27]. Despite their practicability, classical gain-scheduling designs do not offer any guarantees regarding robustness, performance or stability of the overall nonlinear system [28]. By the socalled LPV framework the disadvantages of classical gain scheduling can be overcome. An overview of application results of LPV control for different kind of applications can be found in [29]. The LPV approach has been successfully applied to an electromagnetic actuator [30] and a ball screw drive [31], among others.

This paper is based on the previous work [32], where SEs are used for joint-side velocity feedback to damp vibrations drive-based. However, the linear controller design did not take into account the nonlinear robot dynamics. The consequence is a conservative controller design that does not guarantee performance and stability for different manipulator configurations. The main contribution of this paper is to extend this approach using a LPV-based gain-scheduled control design. Furthermore, the approach is enhanced with the one presented in [26], where an optimization-based design of a robust P-PI with SEs for the position control loop was proposed. We show that the control approach and its inherent robustness improve the dynamic path accuracy of IRs with heavy payload despite uncertain drivetrain dynamics and under variation of the manipulator configuration. It should be noted, that, in order to 


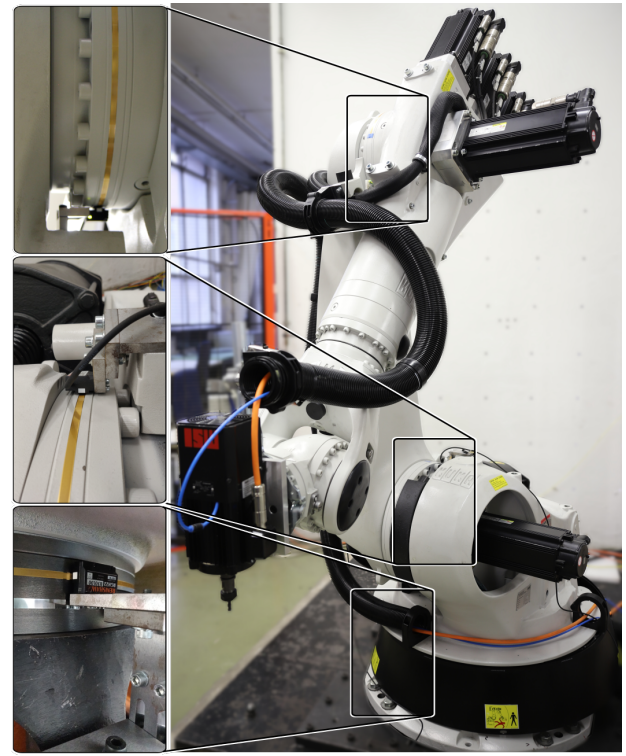

Fig. 1. KUKA KR210-2 IR testbench with SEs.

achieve a high dynamic path accuracy, the presented feedback controller should be combined with appropriate feedforward control [9] and trajectory generation [11].

The paper is organized as follows. Section II describes the experimental setup, derives the LPV model and provides an identification method. Based on this, in Section III the proposed gain-scheduled control design is introduced. The controller synthesis as well as experimental results are presented in Section IV. Finally, summarizing remarks are given in Section V.

\section{Modeling of Industrial Robots}

From a practical control design perspective, a simple model that describes the system within specified error bounds, while being identifiable with reasonable effort, is often preferred. Therefore, in this section, a LPV model of an IR as well as an identification method is proposed.

\section{A. Experimental Setup}

The testbench with the KUKA KR210-2 with standard IR kinematics used for the experimental investigations is shown in Fig. 1. The manipulator has $N=6$ revolute joints and a nominal payload of $210 \mathrm{~kg}$. Cycloidal drives from Nabtesco are installed in the robot joints, causing the dominant elasticity to lie in the axis of rotation of the joints. The three base joints are equipped with SEs, which provide a resolution of the joint position of about $1 \mu \mathrm{rad}$. Moreover, the testbench is operated by a self-developed open control platform based on the real-time operating system INtime of TenAsys. This platform allows the implementation of almost arbitrary controller structures. All applications running on this platform are developed in $\mathrm{C}++$. A more detailed description of the experimental setup is given in [26].

\section{B. Quasi-LPV Model of Industrial Robot}

The derivation of the LPV model originates from the well-known dynamics model of a serial, flexible joint robot according to [6], which is extended by the dissipation effects of motor-side friction and linear joint damping. It denotes as follows

$$
\begin{aligned}
M(\boldsymbol{q}) \ddot{\boldsymbol{q}}+\boldsymbol{C}(\boldsymbol{q}, \dot{\boldsymbol{q}}) \dot{\boldsymbol{q}}+\boldsymbol{g}(\boldsymbol{q})+\boldsymbol{J}^{\mathrm{T}}(\boldsymbol{q}) \boldsymbol{h}_{\mathrm{e}} & =\tau_{\mathrm{g}} \\
\boldsymbol{J}_{\mathrm{m}} \cdot \ddot{\boldsymbol{\theta}}+\tau_{f}+\boldsymbol{U}^{-1} \cdot \tau_{\mathrm{g}} & =\tau_{\mathrm{m}} \\
\boldsymbol{K} \cdot\left(\boldsymbol{U}^{-1} \boldsymbol{\theta}-\boldsymbol{q}\right)+\boldsymbol{D} \cdot\left(\boldsymbol{U}^{-1} \dot{\boldsymbol{\theta}}-\dot{\boldsymbol{q}}\right) & =\tau_{\mathrm{g}} \\
\boldsymbol{F}_{\mathrm{c}} \cdot \operatorname{sign}\left(\boldsymbol{U}^{-1} \dot{\boldsymbol{\theta}}\right)+\boldsymbol{F}_{\mathbf{v}} \cdot \boldsymbol{U}^{-1} \dot{\boldsymbol{\theta}} & =\tau_{\boldsymbol{f}},
\end{aligned}
$$

with the generalized coordinates of the motor positions $\boldsymbol{\theta} \in \mathbb{R}^{N}$ and joint positions $\boldsymbol{q} \in \mathbb{R}^{N}$. Moreover, the rigid body dynamics denoting the inertia matrix $M(q)$, the gravity vector $\boldsymbol{g}(\boldsymbol{q})$ as well as the Coriolis and centrifugal forces $\boldsymbol{C}(\boldsymbol{q}, \dot{\boldsymbol{q}})$ are included. The impact of the generalized contact forces of the tool center point (TCP) with the environment is given by $\boldsymbol{J}^{\mathbf{T}}(\boldsymbol{q}) \boldsymbol{h}_{\mathrm{e}}$. The drivetrain dynamics are coupled to the rigid body dynamics via the diagonal gear ratio matrix $\boldsymbol{U}$, the diagonal joint stiffness matrix $\boldsymbol{K}$ and the diagonal damping matrix $D$. The drivetrain dynamics consist of the motor-side friction $\tau_{f}$, the motor torques $\tau_{\mathbf{m}}$ and the diagonal motor inertia matrix $\boldsymbol{J}_{\mathrm{m}}$. The motor-side friction $\boldsymbol{\tau}_{\boldsymbol{f}}$ is modeled by the diagonal viscous $\boldsymbol{F}_{\mathbf{v}}$ and diagonal Coulomb friction force matrix $\boldsymbol{F}_{\mathbf{c}}$.

We consider a standard LPV system in state space representation [33]

$$
\begin{aligned}
& \dot{\boldsymbol{x}}=\boldsymbol{A}(\boldsymbol{\rho}(t)) \boldsymbol{x}+\boldsymbol{B}(\boldsymbol{\rho}(t)) \boldsymbol{u} \\
& \boldsymbol{y}=\boldsymbol{C}(\boldsymbol{\rho}(t)) \boldsymbol{x}+\boldsymbol{D}(\boldsymbol{\rho}(t)) \boldsymbol{u}
\end{aligned}
$$

with the system matrix $\boldsymbol{A}$, the input matrix $\boldsymbol{B}$, the output matrix $C$, the feedthrough matrix $D$ and the exogenous scheduling vector $\boldsymbol{\rho}$. This representation is equivalent to the input-output form

$$
\boldsymbol{G}(s, \boldsymbol{\rho})=\boldsymbol{C}(\boldsymbol{\rho})(s \boldsymbol{I}-\boldsymbol{A}(\boldsymbol{\rho}))^{-1} \boldsymbol{B}(\boldsymbol{\rho})+\boldsymbol{D}(\boldsymbol{\rho}) .
$$

To formulate the robot model in the LPV framework, the dynamics model (1) is treated as $i=1, \ldots, N$ independent SISO systems

$$
\begin{aligned}
M_{i i}{ }^{-1}(\boldsymbol{q}) \cdot\left(\tau_{\mathrm{g}, i}-\tau_{\mathrm{ext}, i}\right) & =\ddot{q}_{i} \\
J_{\mathrm{m}, i}{ }^{-1} \cdot\left(\tau_{\mathrm{m}, i}-\tau_{\mathrm{f}, i}-u_{i}{ }^{-1} \tau_{\mathrm{g}, i}\right) & =\ddot{\theta}_{i} \\
k_{i} \cdot\left(u_{i}{ }^{-1} \theta_{i}-q_{i}\right)+d_{i} \cdot\left(u_{i}{ }^{-1} \dot{\theta}_{i}-\dot{q}_{i}\right) & =\tau_{\mathrm{g}, i},
\end{aligned}
$$

which are controlled on joint level, as it is common for the P-PI [10], with the exception of the diagonal mass matrix elements $M_{i i}(\boldsymbol{q})$. The residual rigid body dynamics are considered as disturbance

$$
\begin{aligned}
\tau_{\text {ext }}=J^{\mathrm{T}}(q) h_{\mathrm{e}} & +g(q)+C(q, \dot{q}) \dot{q} \\
+ & {[M(q)-\operatorname{diag}(M(q))] \ddot{q}, }
\end{aligned}
$$

where $\tau_{\text {ext, } i}$ is the $i$-th element of the disturbance $\tau_{\text {ext }} \in \mathbb{R}^{N}$. The entries of the matrices of rigid body dynamics are constants or trigonometric functions of the joint positions and therefore Lipschitz continuous [26]. Furthermore, it is assumed, that the generalized contact forces of the TCP with 
the environment $\boldsymbol{J}^{\mathbf{T}}(\boldsymbol{q}) \boldsymbol{h}_{\mathrm{e}}$ are bounded. Consequently, the disturbance $\tau_{\text {ext }, i}$ is bounded, too.

Closed-loop torque control is implemented for each joint, whereby its time constant is more than an order of magnitude lower than that of the mechanics [26]. The transfer function from the desired motor torque $\tau_{\mathrm{m}, \mathrm{d}, i}$ to the motor torque $\tau_{\mathrm{m}, i}$

$$
G_{\tau_{\mathrm{m}, i}}=\frac{\tau_{\mathrm{m}, i}}{\tau_{\mathrm{m}, \mathrm{d}, i}}=\frac{1}{1+s \cdot T_{\tau, i}}
$$

is approximated by a first order lag element and assigned to the plant dynamics.

The inverse of the diagonal mass matrix elements is introduced as scheduling signal

$$
\tilde{\rho}_{i}=\frac{1}{M_{i i}(\boldsymbol{q})} \in\left[\frac{1}{M_{i, \max }}, \frac{1}{M_{i, \min }}\right] \in \mathbb{R}^{1}
$$

per each joint $i$. The transfer function from the motor torque $\tau_{\mathrm{m}, i}$ to the motor velocity $\dot{\theta}_{i}$

$$
\begin{aligned}
G_{\dot{\theta}_{i}} & =\left.\frac{\dot{\theta}_{i}}{\tau_{\mathrm{m}, i}}\right|_{\tau_{\mathrm{f}, i}, \tau_{\mathrm{ext}, i}=0} \\
& =\frac{1}{s} \cdot \frac{\frac{1}{J_{\mathrm{m}, i}} s^{2}+\tilde{\rho}_{i} \frac{d_{i}}{J_{\mathrm{m}, i}} s+\tilde{\rho}_{i} \frac{k_{i}}{J_{\mathrm{m}, i}}}{s^{2}+\left(\tilde{\rho}_{i}+\frac{1}{u_{i}{ }^{2} J_{\mathrm{m}, i}}\right) d_{i} s+\left(\tilde{\rho}_{i}+\frac{1}{u_{i}{ }^{2} J_{\mathrm{m}, i}}\right) k_{i}}
\end{aligned}
$$

as well as the transfer function from the motor velocity $\dot{\theta}_{i}$ to the joint velocity $\dot{q}_{i}$

$$
G_{\dot{q}_{i}}=\left.\frac{\dot{q}_{i}}{\dot{\theta}_{i}}\right|_{\tau_{\mathrm{ext}, i}=0}=\frac{1}{u_{i}} \frac{\tilde{\rho}_{i} d_{i} s+\tilde{\rho}_{i} k_{i}}{s^{2}+\tilde{\rho}_{i} d_{i} s+\tilde{\rho}_{i} k_{i}}
$$

result. Furthermore, the transfer function from the joint velocity $\dot{q}_{i}$ to the joint position $q_{i}$

$$
G_{q_{i}}=\frac{q_{i}}{\dot{q}_{i}}=\frac{1}{s}
$$

yields as simple integrator. Based on these derivations, the closed-loop motor-side velocity control loop, which is also assigned to the plant dynamics, is given by

$$
G_{\dot{\theta}_{i}, \mathrm{CL}}=\left.\frac{\dot{\theta}_{i}}{\dot{\theta}_{\mathrm{d}, i}}\right|_{\tau_{\mathrm{f}, i}, \tau_{\mathrm{ext}, i}=0}=\frac{G_{\dot{\theta}_{i}} G_{\tau_{\mathrm{m}, i}} K_{\mathrm{p}, i} \frac{T_{\mathrm{n}, i} s+1}{T_{\mathrm{n}, i} s}}{1+G_{\dot{\theta}_{i}} G_{\tau_{\mathrm{m}, i}} K_{\mathrm{p}, i} \frac{T_{\mathrm{n}, i} s+1}{T_{\mathrm{n}, i} s}},
$$

with the gains $K_{\mathrm{p}, i}$ and $T_{\mathrm{n}, i}$ of the proportional integral velocity controller. To normalize the scheduling signal $\tilde{\rho}_{i}$ to the range $\rho_{i} \in[-1,1]$ with zero mean value, the transformation

$$
\rho_{i}=\frac{1}{d_{\rho_{i}}}\left(\tilde{\rho}_{i}-\mu_{\rho_{i}}\right), d_{\rho_{i}}=\frac{\overline{\tilde{\rho}_{i}}-\underline{\tilde{\rho}_{i}}}{2}, \mu_{\rho_{i}}=\frac{\overline{\tilde{\rho}_{i}}+\underline{\tilde{\rho}_{i}}}{2}
$$

with the absolute distance $d_{\rho_{i}}$ and the mean $\mu_{\rho_{i}}$ as well as the

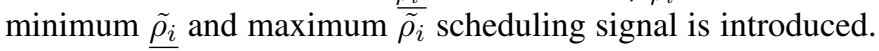
This results in the LPV model

$$
\boldsymbol{G}\left(s, \rho_{i}\right)=\left[\begin{array}{cc}
G_{q_{i}} & 0 \\
0 & G_{\dot{q}_{i}} G_{\dot{\theta}_{i}, \mathrm{CL}}
\end{array}\right]
$$

with the input vector $\boldsymbol{u}$ and output vector $\boldsymbol{y}$

$$
\boldsymbol{u}=\left[\begin{array}{c}
\dot{q}_{i} \\
\dot{\theta}_{\mathrm{d}, i}
\end{array}\right], \boldsymbol{y}=\left[\begin{array}{c}
q_{i} \\
\dot{q}_{i}
\end{array}\right] \text {. }
$$

This model is a quasi-LPV model, since the scheduling variable depends on the system state. It should be noted that the timescale of the mass matrix (inverse of the scheduling signal) is considered to be significantly longer than that of the joint dynamics (process to be controlled), which is typical for industrial robots [18]. Therefore, although the system is not LPV in a strict sense, it can be treated accordingly.

\section{System Identification}

Since the identification concerns a quasi-LPV model, it is performed based on the nonlinear model (1). The identification of the rigid body parameters (masses, center of masses and moments of inertia) of the manipulator is conducted using the method [34] of reformulating the nonlinear dynamics as a model

$$
\begin{aligned}
\tau_{\mathrm{s}} & =M(\boldsymbol{q}) \ddot{\boldsymbol{q}}+\boldsymbol{C}(\boldsymbol{q}, \dot{\boldsymbol{q}}) \dot{\boldsymbol{q}}+\boldsymbol{g}(\boldsymbol{q}) \\
& =\boldsymbol{Y}_{\mathrm{s}}(\boldsymbol{q}, \dot{\boldsymbol{q}}, \ddot{\boldsymbol{q}}) \cdot \boldsymbol{\pi}_{\mathrm{s}} \\
& =\boldsymbol{U}\left(\boldsymbol{\tau}_{\mathrm{m}}-\boldsymbol{J}_{\mathrm{m}} \ddot{\boldsymbol{q}}-\tau_{\boldsymbol{f}}(\dot{\boldsymbol{q}})\right),
\end{aligned}
$$

which is linear in the model parameter $\boldsymbol{\pi}_{\mathbf{s}}$. The excitation trajectories are generated on the basis of Fourier series with a defined frequency range [35], where the Fourier coefficients are determined by minimizing the condition number of the regressor matrix $\boldsymbol{Y}_{\mathbf{s}}(\boldsymbol{q}, \dot{\boldsymbol{q}}, \ddot{\boldsymbol{q}})$. This method ensures a periodic, bandwidth-limited excitation of the system. The maximum frequency of the excitation trajectories is chosen to be smaller than the lowest eigenfrequency of the joints to separate the identification of the rigid body parameters can from joint elasticity effects $\left(\boldsymbol{q} \approx \boldsymbol{U}^{-1} \dot{\boldsymbol{\theta}}\right)$. In Fig. 2 , the measured motor torques $\tau_{\mathbf{m}}$ for such generated trajectories as well as the motor torques determined by least squares estimation are shown.

The regressor matrix of the rigid body dynamics $\boldsymbol{Y}_{\mathbf{s}}(\boldsymbol{q}, \dot{\boldsymbol{q}}, \ddot{\boldsymbol{q}})$ is still linear in joint acceleration $\ddot{\boldsymbol{q}}$, which allows to split the regressor matrix

$$
\tau_{\mathrm{s}}=\boldsymbol{Y}_{\mathbf{s}}(\boldsymbol{q}, \dot{\boldsymbol{q}}, \ddot{\boldsymbol{q}}) \cdot \boldsymbol{\pi}_{\mathrm{s}}=\boldsymbol{Y}_{\mathrm{s}, \mathrm{A}}\left(\boldsymbol{q}, \boldsymbol{\pi}_{\mathrm{s}}\right) \cdot \ddot{\boldsymbol{q}}+\boldsymbol{Y}_{\mathrm{s}, \mathrm{B}}\left(\boldsymbol{q}, \dot{\boldsymbol{q}}, \boldsymbol{\pi}_{\mathrm{s}}\right)
$$

Hence, the matrix $\boldsymbol{Y}_{\mathbf{s}, \mathrm{A}}\left(\boldsymbol{q}, \boldsymbol{\pi}_{\mathbf{s}}\right)$ corresponds to the mass matrix $\boldsymbol{Y}_{\mathbf{s}, \mathrm{A}}\left(\boldsymbol{q}, \boldsymbol{\pi}_{\mathrm{s}}\right)=\boldsymbol{M}(\boldsymbol{q})$ and the required diagonal elements of the mass matrix are obtained, which are shown in Fig. 3.

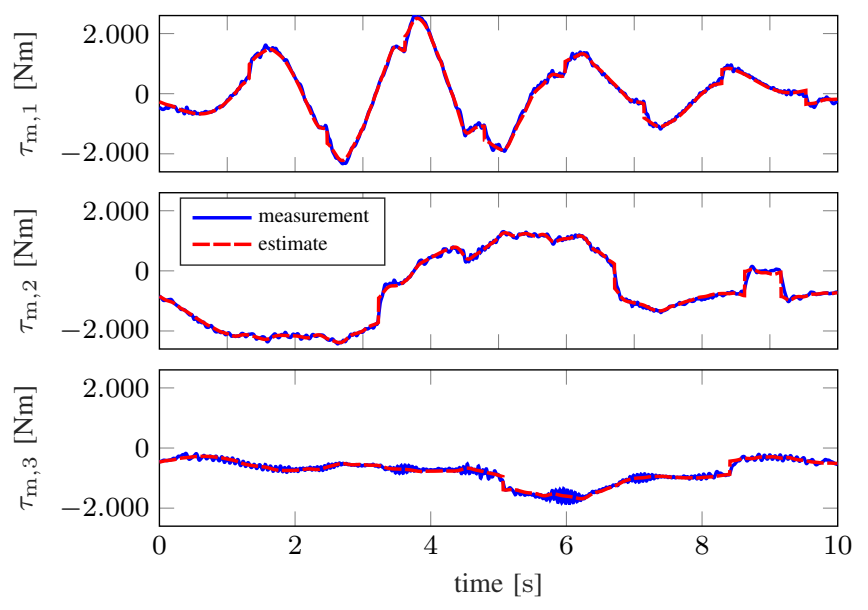

Fig. 2. Measured and identified motor torque of the first three joints of the KUKA KR210 of the rigid body dynamics identification. 


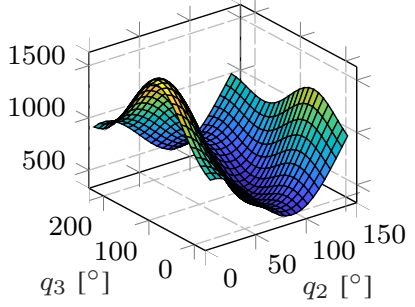

(a) $M_{11}\left(\mathrm{kgm}^{2}\right)$

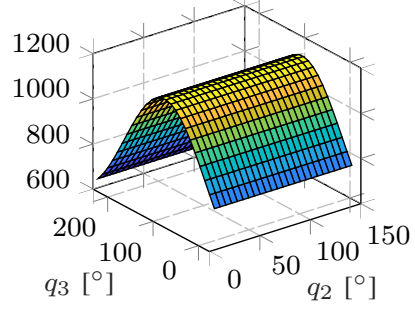

(b) $M_{22}\left(\mathrm{kgm}^{2}\right)$
Fig. 3. Identified diagonal mass matrix elements $\left(M_{33} \approx 243.9 \mathrm{kgm}^{2}\right)$.

TABLE I

IDENTIFIED ELASTIC PARAMETERS OF THE KUKA KR210-2

\begin{tabular}{cllll}
\hline Parameter & Joint 1 & Joint 2 & Joint 3 & Unit \\
\hline$k_{i}$ & $3.0 \mathrm{e} 6$ & $3.0 \mathrm{e} 6$ & $8.2 \mathrm{e} 6$ & $\mathrm{Nm} / \mathrm{rad}$ \\
$d_{i}$ & $4.6 \mathrm{e} 3$ & $3.8 \mathrm{e} 3$ & $5.8 \mathrm{e} 3$ & $\mathrm{Nms} / \mathrm{rad}$ \\
\hline
\end{tabular}

The identification of the joint elasticity is carried out with closed-loop input-output experiments, while using a motorside P-PI under a rigid body assumption [10]. Each joint is separately excited by a logarithmic chirp of the motor position with an amplitude of $4 \mathrm{e}-3^{\circ}$, a frequency range of $1 \mathrm{~Hz}$ to $35 \mathrm{~Hz}$ and an offset velocity of $2 \%$ s. The joint stiffness and damping are estimated by minimizing the mean squared error between the measurement and the model (9) in the frequency domain using the Levenberg-Marquardt algorithm. Fig. 4 shows the corresponding Bode magnitude plots as well as the estimated frequency responses. The estimated frequency responses capture the transfer behavior up to the first eigenfrequency. The uncertainty at higher frequency is tolerated achieving a trade-off between complexity and model accuracy. The obtained elastic parameters are listed in Table I.

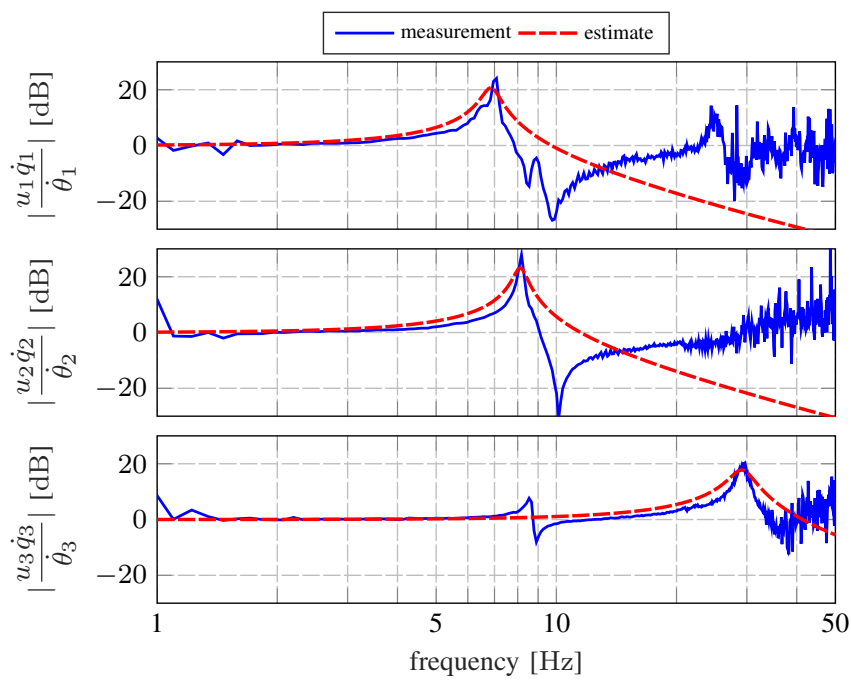

Fig. 4. Comparison of the magnitude frequency response of the first three joints of the KUKA KR210-2 (blue) and the identified joint model (red) (input: $u_{i}{ }^{-1} \dot{\theta}_{i}$, output: $\dot{q}_{i}$ ).

\section{GAIN-SCHEDULED CONTROL}

The LPV theory allows extending a linear controller design to adapt it to the time-varying dynamics of a special class of nonlinear system. Thereby, well-understood linear design tools can be used for a nonlinear controller design. Thus, the main disadvantage of classical gain scheduling of a missing stability analysis over the entire parameter range is overcome.

\section{A. LPV Controller Design}

To carry out the controller design according to [33], the control loop is transformed into the structure shown in Fig. 5. Therefore, the LPV plant (2) is extended by the external disturbance input $\boldsymbol{w}$ and the performance variable $\boldsymbol{z}$

$$
\begin{aligned}
\dot{\boldsymbol{x}} & =\boldsymbol{A}(\boldsymbol{\rho}) \boldsymbol{x}+\boldsymbol{B}_{1}(\boldsymbol{\rho}) \boldsymbol{w}+\boldsymbol{B}_{2}(\boldsymbol{\rho}) \boldsymbol{u} \\
\boldsymbol{z} & =\boldsymbol{C}_{1}(\boldsymbol{\rho}) \boldsymbol{x}+\boldsymbol{D}_{11}(\boldsymbol{\rho}) \boldsymbol{w}+\boldsymbol{D}_{12}(\boldsymbol{\rho}) \boldsymbol{u} \\
\boldsymbol{e} & =\boldsymbol{C}_{2}(\boldsymbol{\rho}) \boldsymbol{x}+\boldsymbol{D}_{21}(\boldsymbol{\rho}) \boldsymbol{w}+\boldsymbol{D}_{22}(\boldsymbol{\rho}) \boldsymbol{u},
\end{aligned}
$$

with the state $\boldsymbol{x}$, the input $\boldsymbol{u}$ and the control error $\boldsymbol{e}$, as usual in robust control theory [36]. It is further assumed that the system matrices depend affinely on the scheduling signal $\rho$. Subsequently, a LPV controller $\boldsymbol{K}$ in state space representation

$$
\begin{aligned}
\dot{x}_{K} & =A_{K}(\rho) x_{K}+B_{K}(\rho) e \\
u & =C_{K}(\rho) x_{K}+D_{K}(\rho) e
\end{aligned}
$$

is applied. When closing the control loop, the affinity of the whole system is not maintained inherently. In [33] it was found out, that the matrix $\boldsymbol{D}_{22}(\boldsymbol{\rho})$ has to equal zero as well as the matrices $\boldsymbol{B}_{2}, \boldsymbol{C}_{2}, \boldsymbol{D}_{12}$ and $\boldsymbol{D}_{21}$ have to be parameter independent. Otherwise, filter methods found in [33] may be applied. The resulting controller equals a family of linear controllers, which are gain-scheduled in a linear manner regarding the scheduling signal $\rho \in \mathbb{R}^{m}$. Regarding an affine dependency, the LPV controller corresponds to a linear interpolation of the linear controllers at the $r=2^{m}$ vertices of the parameter space. It results the LPV controller $\Omega(\rho)$ in state space representation

$$
\boldsymbol{\Omega}(\boldsymbol{\rho})=\sum_{j=1}^{r} \alpha_{j} \boldsymbol{\Omega}_{j}=\sum_{j=1}^{r} \alpha_{j}\left[\begin{array}{ll}
\boldsymbol{A}_{\boldsymbol{K}, j} & \boldsymbol{B}_{\boldsymbol{K}, j} \\
\boldsymbol{C}_{\boldsymbol{K}, j} & \boldsymbol{D}_{\boldsymbol{K}, j}
\end{array}\right]
$$

with $\alpha_{j} \geq 0$ and $\sum_{j=1}^{r} \alpha_{j}=1$.

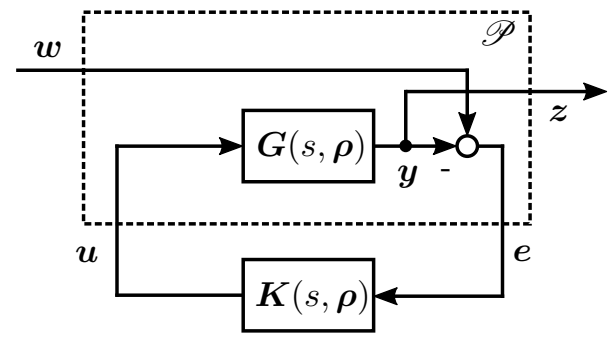

Fig. 5. Structure for the LPV controller design (based on [33]). 


\section{B. Stability Analysis}

For a control loop with LPV structure, a systematic stability analysis can be performed based on Lyapunov's second method [37]. This avoids the assumption of a slowly varying scheduling signal that is typical for classical gain scheduling. A LPV-system

$$
\dot{\boldsymbol{x}}=\boldsymbol{A}(\rho) \boldsymbol{x}
$$

is quadratically stable [38], if there exists a positive definite Lyapunov function

$$
V(\boldsymbol{x})=\boldsymbol{x}^{\top} \boldsymbol{P}_{\mathrm{Q}} \boldsymbol{x},
$$

such that for all trajectories of the scheduling signal the inequality

$$
\boldsymbol{P}_{\mathrm{Q}} \boldsymbol{A}(\boldsymbol{\rho})+\boldsymbol{A}(\boldsymbol{\rho})^{\top} \boldsymbol{P}_{\mathrm{Q}}<0
$$

holds. Due to the constant matrix $\boldsymbol{P}_{\mathrm{Q}}$, the quadratic stability test is valid for arbitrary scheduling signal rates, what is potentially conservative. This conservatism can be reduced using an affine, parameter dependent Lyapunov function

$$
V(\boldsymbol{x}, \boldsymbol{\rho})=\boldsymbol{x}^{\top} \boldsymbol{P}(\boldsymbol{\rho}) \boldsymbol{x} .
$$

A LPV system is affinely quadratically stable [39], if

$$
\begin{array}{r}
\boldsymbol{P}(\boldsymbol{\rho})=\boldsymbol{P}_{0}+\boldsymbol{P}_{1} \rho_{1}+\ldots+\boldsymbol{P}_{k} \rho_{k}>0 \\
\boldsymbol{P}(\boldsymbol{\rho}) \boldsymbol{A}(\boldsymbol{\rho})+\boldsymbol{A}(\boldsymbol{\rho})^{\top} \boldsymbol{P}(\boldsymbol{\rho})+\boldsymbol{P}(\dot{\boldsymbol{\rho}})-\boldsymbol{P}_{0}<0
\end{array}
$$

holds for symmetric matrices $\boldsymbol{P}_{0}, \ldots, \boldsymbol{P}_{k}$.

Both stability tests can be reduced to linear matrix inequality problems and thus be solved numerically efficiently. The Robust Control Toolbox of MATLAB [40] provides appropriate functionalities to test for quadratic (quadstab) and affine quadratic stability (pdlstab) of LPV systems using linear matrix inequalities. It should be noted, however, that the analyses guarantee stability against the LPV model and not against the original nonlinear system.

\section{LPV Damping Control}

The proposed linear drive-based damping control law

$$
\begin{aligned}
\dot{\theta}_{\mathrm{d}, i}=u_{i}\left[K_{\mathrm{v}, i}\left(q_{\mathrm{d}, i}-q_{i}\right)\right. & -\frac{K_{\mathrm{d}, i} d_{i} \cdot s+K_{\mathrm{k}, i} k_{i}}{d_{i} \cdot s+k_{i}} \cdot \dot{q}_{i} \\
& \left.+\left(1+K_{\mathrm{k}, i}\right) \cdot \dot{q}_{\mathrm{d}, i}\right]
\end{aligned}
$$

consists of a proportional controller of the joint position $q_{i}$ with the gain $K_{\mathrm{v}, i}$, a dynamic feedback with the stiffness gain $K_{\mathrm{k}, i}$ and the damping gain $K_{\mathrm{d}, i}$ based on the joint velocity $\dot{q}_{i}$ and a feedforward term to account for a potential steady-state error during constant velocity trajectories. The corresponding block diagram of the LPV plant, including the control law, is shown in Fig. 6. The analytical derivation of the linear damping control law can be found in [32].

In the following, this linear control law is extended to an adaptive controller by the LPV theory. To obtain the structure of Fig. 5, the controller (25), without the feedforward term, is converted to the state space representation

$$
\begin{aligned}
\dot{\boldsymbol{x}}_{\boldsymbol{K}} & =\left[-\frac{k_{i}}{d_{i}}\right] \boldsymbol{x}_{\boldsymbol{K}}+\left[\begin{array}{ll}
0 & 1
\end{array}\right] \boldsymbol{e} \\
\boldsymbol{u} & =\left[\begin{array}{c}
0 \\
\left(K_{\mathrm{d}, i}\left(\rho_{i}\right)-K_{\mathrm{k}, i}\left(\rho_{i}\right)\right) \frac{k_{i}}{d_{i}}
\end{array}\right] \boldsymbol{x}_{\boldsymbol{K}} \\
& +\left[\begin{array}{cc}
0 & 1 \\
K_{\mathrm{v}, i}\left(\rho_{i}\right) & -K_{\mathrm{d}, i}\left(\rho_{i}\right)
\end{array}\right] \boldsymbol{e}
\end{aligned}
$$

with the external disturbance input $\boldsymbol{w}$, the control error $\boldsymbol{e}$ and the output vector $\boldsymbol{y}$ to

$$
\boldsymbol{w}=\left[\begin{array}{c}
q_{\mathrm{d}, i} \\
0
\end{array}\right], \boldsymbol{e}=\left[\begin{array}{c}
q_{\mathrm{d}, i}-q_{i} \\
-\dot{q}_{i}
\end{array}\right], \boldsymbol{y}=\boldsymbol{z} .
$$

To preserve the affinity of the system despite the parameter dependency of the matrix $C_{2}$, the post-filtering

$$
\begin{aligned}
\dot{\boldsymbol{x}}_{\mathrm{e}} & =\left[\begin{array}{cc}
-\lambda_{1} & 0 \\
0 & -\lambda_{2}
\end{array}\right] \boldsymbol{x}_{\mathrm{e}}+\left[\begin{array}{cc}
\lambda_{1} & 0 \\
0 & \lambda_{2}
\end{array}\right] \boldsymbol{e} \\
\tilde{\boldsymbol{e}} & =\left[\begin{array}{ll}
1 & 0 \\
0 & 1
\end{array}\right] \boldsymbol{x}_{\mathrm{e}}
\end{aligned}
$$

with the eigenvalues $\lambda_{1}$ and $\lambda_{2}$ is introduced, where the filter bandwidth is chosen larger than the fastest system dynamics. Since the joint position $q_{i}$ is decisive for the overall performance, the performance output

$$
\tilde{z}=\left[\begin{array}{ll}
1 & 0
\end{array}\right] \boldsymbol{z}
$$

and the external disturbance input

$$
\tilde{w}=\left[\begin{array}{ll}
1 & 0
\end{array}\right] \boldsymbol{w}
$$

is reduced accordingly. Assuming an ideal motor velocity control loop $\dot{\theta}_{i} / \dot{\theta}_{\mathrm{d}, i} \approx 1$, the closed-loop system

$$
\begin{aligned}
\dot{\boldsymbol{x}}_{\mathrm{CL}} & =\boldsymbol{A}_{\mathrm{CL}}\left(\rho_{i}\right) \boldsymbol{x}_{\mathrm{CL}}+\boldsymbol{B}_{\mathrm{CL}}\left(\rho_{i}\right) \tilde{w} \\
\tilde{z} & =\boldsymbol{C}_{\mathrm{CL}}\left(\rho_{i}\right) \boldsymbol{x}_{\mathrm{CL}}
\end{aligned}
$$

with the system matrix $\boldsymbol{A}_{\mathrm{CL}}$, shown in (32) at the top of the next page, the input

$$
\boldsymbol{B}_{\mathrm{CL}}{ }^{\top}=\left[\begin{array}{llllll}
0 & 0 & 0 & \lambda_{1} & 0 & 0
\end{array}\right]^{\top}
$$

and the output

$$
\boldsymbol{C}_{\mathrm{CL}}=\left[\begin{array}{llllll}
1 & 0 & 0 & 0 & 0 & 0
\end{array}\right] .
$$

yields.

The linear controllers at the vertices of the parameter space are designed via a robust $H_{\infty}$ synthesis. Using the frequency responses $\tilde{G}\left(j \omega, \rho_{i}\right)$ obtained during system identification, a multiplicative uncertainty model

$$
\Delta_{\mathrm{m}}\left(j \omega, \rho_{i}\right)=\frac{\tilde{G}\left(j \omega, \rho_{i}\right)-G\left(j \omega, \rho_{i}\right)}{G\left(j \omega, \rho_{i}\right)},
$$

of the nominal plant $G\left(j \omega, \rho_{i}\right)$ is determined. To guarantee robust stability, the weighting function $W_{\mathrm{T}}\left(j \omega, \rho_{i}\right)$

$$
\left|W_{\mathrm{T}}\left(j \omega, \rho_{i}\right)\right| \geq\left|\Delta_{\mathrm{m}}\left(j \omega, \rho_{i}\right)\right|, \forall \omega \text { and } \forall, \rho_{i}
$$

has to be chosen larger than the multiplicative uncertainty $\Delta_{\mathrm{m}}\left(j \omega, \rho_{i}\right)$ for all frequencies $\omega$ and every value of the scheduling signal $\rho_{i}$ [36]. 


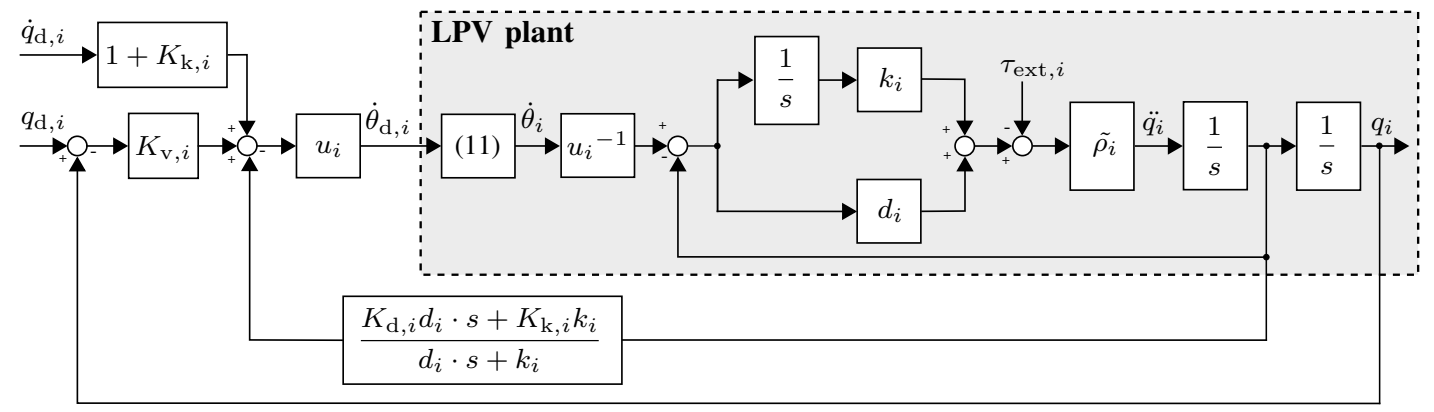

Fig. 6. Block diagram of the LPV plant including the drive-based damping control.

$$
\boldsymbol{A}_{\mathrm{CL}}=\left[\begin{array}{cccccc}
0 & 0 & 0 & 1 & 0 & 0 \\
0 & 0 & 1 & 0 & 0 & 0 \\
0 & -k_{i}\left(\mu_{\rho_{i}}+d_{\rho_{i}} \rho_{i}\right) & -d_{i}\left(\mu_{\rho_{i}}+d_{\rho_{i}} \rho_{i}\right) & K_{\mathrm{v}, i}\left(\rho_{i}\right) & -K_{\mathrm{d}, i}\left(\rho_{i}\right) & \frac{k_{i}}{d_{i}}\left(K_{\mathrm{d}, i}\left(\rho_{i}\right)-K_{\mathrm{k}, i}\left(\rho_{i}\right)\right) \\
-\lambda_{1} & 0 & 0 & -\lambda_{1} & 0 & 0 \\
0 & \lambda_{2} k_{i}\left(\mu_{\rho_{i}}+d_{\rho_{i}} \rho_{i}\right) & \lambda_{2} d_{i}\left(\mu_{\rho_{i}}+d_{\rho_{i}} \rho_{i}\right) & 0 & -\lambda_{2} & 0 \\
0 & 0 & 0 & 0 & 1 & -\frac{k_{i}}{d_{i}}
\end{array}\right]
$$

Therefore, the transfer function from the desired joint velocity $\dot{q}_{\mathrm{d}, i}$ to the joint velocity $\dot{q}_{i}$ is weighted by

$$
W_{\mathrm{T}, \dot{q}_{i}}\left(s, \rho_{i}\right)=D_{\mathrm{d}, \dot{\mathrm{q}}_{\mathrm{i}}} \frac{s^{2}+2 \xi \epsilon_{1} \omega_{0} s+\left(\epsilon_{1} \omega_{0}\right)^{2}}{\left(\epsilon_{1} \omega_{0}\right)^{2}}
$$

as well as the transfer function from the desired joint position $q_{\mathrm{d}, i}$ to the joint position $q_{i}$ by

$$
W_{\mathrm{T}, q_{i}}\left(s, \rho_{i}\right)=\frac{s^{2}+2 \xi \epsilon_{2} \omega_{0} s+\left(\epsilon_{2} \omega_{0}\right)^{2}}{\left(\epsilon_{2} \omega_{0}\right)^{2}}
$$

considering the measured frequency responses (cf. Fig. 4). By means of the parameters $\epsilon_{1}=1.1, \epsilon_{2}=0.75$, the cut-off frequency of the weighting functions is specified as a function of the eigenfrequency $\omega_{0}=\sqrt{k_{i} \rho_{i}}$ and thus of the scheduling signal. Whereas, with the parameter $\xi=0.7$ a well-damped system behavior is specified. For the weighting on the velocity level, an additional degree of freedom is provided via the parameter $D_{\mathrm{d}, \dot{\mathrm{q}}_{\mathrm{i}}}=0.5$ to parameterize the DC gain.

To avoid actuator saturation, the transfer function of the desired joint position $q_{\mathrm{d}, i}$ to the motor torque $\tau_{\mathrm{m}, i}$ is weighted by the gain

$$
W_{\mathrm{KS}, q_{i}}=\left(u_{i} \tau_{\max , i} \kappa\right)^{-1} \text {. }
$$

The gain is chosen based on the maximum motor torque $\tau_{\max , i}$, the gear ratio $u_{i}$ and a user-defined weighting $0<\kappa \leq 1$.

For performance requirements, the sensitivity function of the position control loop from the desired joint position $q_{\mathrm{d}, i}$ to the control error $q_{\mathrm{d}, i}-q_{i}$ is weighted by

$$
W_{\mathrm{S}, q_{i}}=M_{\mathrm{S}, \mathrm{q}_{\mathrm{i}}}^{-1} \frac{s+\lambda \omega_{0}}{s+\omega_{1}}
$$

with the maximum peak of the sensitivity function $M_{\mathrm{S}, \mathrm{q}_{\mathrm{i}}}=$ 1.66 (equals a worst-case gain margin of at least $8 \mathrm{~dB}$ [41]), $\omega_{1}=0.001$ and the performance parameter $\lambda$, which is the only parameter that is iterated during the controller synthesis.

\section{Controller Synthesis AND ExPerimental RESULTS}

The experimental validation is performed with the control architecture shown in Fig. 7. Since only the joint positions are measured via the SEs, but their derivatives are required, the Unscented Kalman Filter (UKF) presented in [32] is used. First, the LTI vertex controllers of the LPV controller (26) are designed according to the $H_{\infty}$ theory. Due to the fixed controller structure, the nonsmooth optimization technique presented in [42] is utilized. This algorithm is commercially available in the robust control toolbox of MATLAB [40]. The gains of the synthesized controllers with a performance parameter $\lambda=0.45$ are listed in Table II. Performed stability tests yield for the second joint quadratic stability over $121.4 \%$ of the parameter space, whereas for the first joint $54.3 \%$ is obtained. Using the less conservative affine quadratic stability test for the first joint, stability is preserved for a maximum scheduling signal rate of $\dot{\rho}_{1} \approx 9.51 / \mathrm{s}$. Under consideration of the identified diagonal mass matrix elements (cf. Fig. 3), this equals maximum joint velocities of $\dot{q}_{2} \approx 112.8 \mathrm{deg} / \mathrm{s}$ and $\dot{q}_{3} \approx 212.3 \mathrm{deg} / \mathrm{s}$, which are larger than the maximum velocities specified by the manufacturer.

To characterize the control performance, the sensitivity function of the position control loop from the desired joint position $q_{\mathrm{d}, i}$ to the control error $e_{i}=q_{\mathrm{d}, i}-q_{i}$ as well as the complementary sensitivity function from the desired joint

TABLE II

LPV CONTROLLER GAINS FOR THE KUKA KR210-2

\begin{tabular}{ccccccccc}
\hline \multirow{2}{*}{ Gain } & \multicolumn{2}{c}{ Joint $\mathbf{1}$} & & \multicolumn{2}{c}{ Joint $\boldsymbol{2}$} & & Joint 3 & \multirow{2}{*}{ Unit } \\
\cline { 2 - 3 } & $\boldsymbol{\Omega}_{1}$ & $\boldsymbol{\Omega}_{2}$ & & $\boldsymbol{\Omega}_{1}$ & $\boldsymbol{\Omega}_{2}$ & & $\boldsymbol{\Omega}_{1}=\boldsymbol{\Omega}_{2}$ & \\
\hline$K_{\mathrm{v}, i}$ & 16.9 & 18.1 & & 19.1 & 19.5 & & 35.5 & $1 / \mathrm{s}$ \\
$K_{\mathrm{k}, i}$ & 0.44 & 0 & & 0.45 & 0.11 & & 0 & - \\
$K_{\mathrm{d}, i}$ & 8.8 & 1.1 & & 10.1 & 6.4 & & 1.09 & - \\
\hline
\end{tabular}




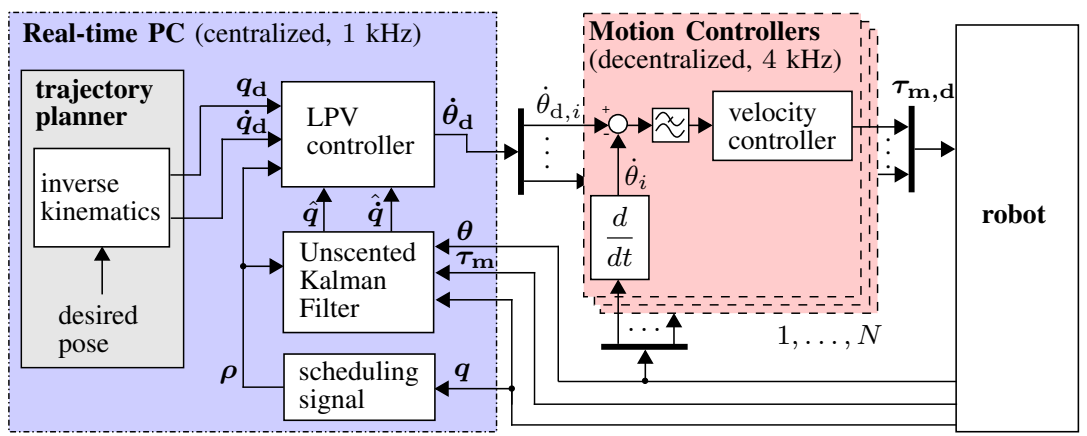

Fig. 7. Block diagram of control architecture for the experimental validation.

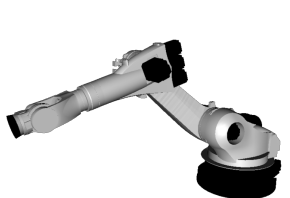

(a)

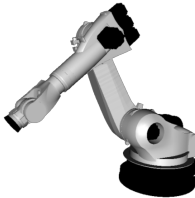

(b)

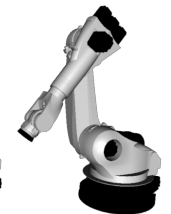

(c)

Fig. 8. Manipulator configurations for experimental validation.

TABLE III

MASS MATRIX ENTRIES OF THE KUKA KR210-2 OF SPECIFIED MANIPULATOR CONFIGURATION (CF. FIG. 8)

\begin{tabular}{cllll}
\hline Configuration & (a) & (b) & (c) & Unit \\
\hline$M_{11}$ & 1371 & 847 & 506 & $\mathrm{kgm}^{2}$ \\
$M_{22}$ & 1019 & 807 & 673 & $\mathrm{kgm}^{2}$ \\
\hline
\end{tabular}

position $q_{\mathrm{d}, i}$ to the joint position $q_{i}$ of the first joint for three different manipulator configurations (cf. Fig. 8 and Table III) are determined (see Fig. 9). Therefore, the system is excited with a random binary signal of the desired motor position $q_{\mathrm{d}, i}$ with an amplitude of $15 \mathrm{e}-3^{\circ}$ and a maximum switching frequency of $100 \mathrm{~Hz}$. The LPV controller achieves good control performance for all three scenarios despite the variation of the manipulator configuration. Moreover, sufficient robustness margins (evident from the maximum peaks of the sensitivity function) are obtained in all cases.

In addition, the control performance in the time domain is investigated by a jerk-limited Cartesian pointto-point trajectory tracking experiment with the initial
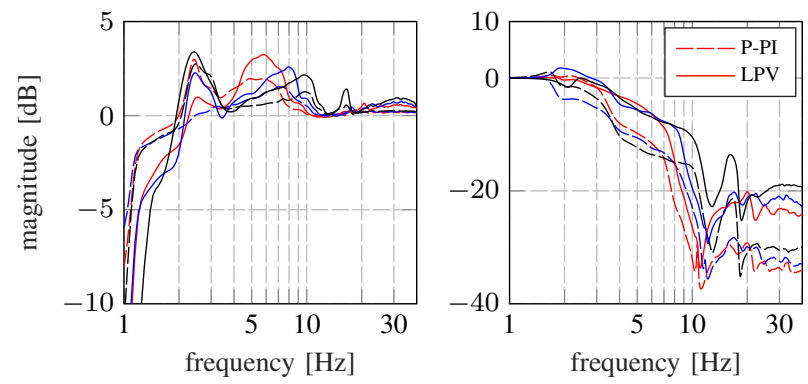

Fig. 9. Sensitivity (left) and complementary sensitivity function (right) of position control loop of the P-PI (dashed) and LPV (solid) controller of the first joint for different manipulator configurations (red: Fig. 8a, blue: Fig. 8b, black: Fig. 8c).
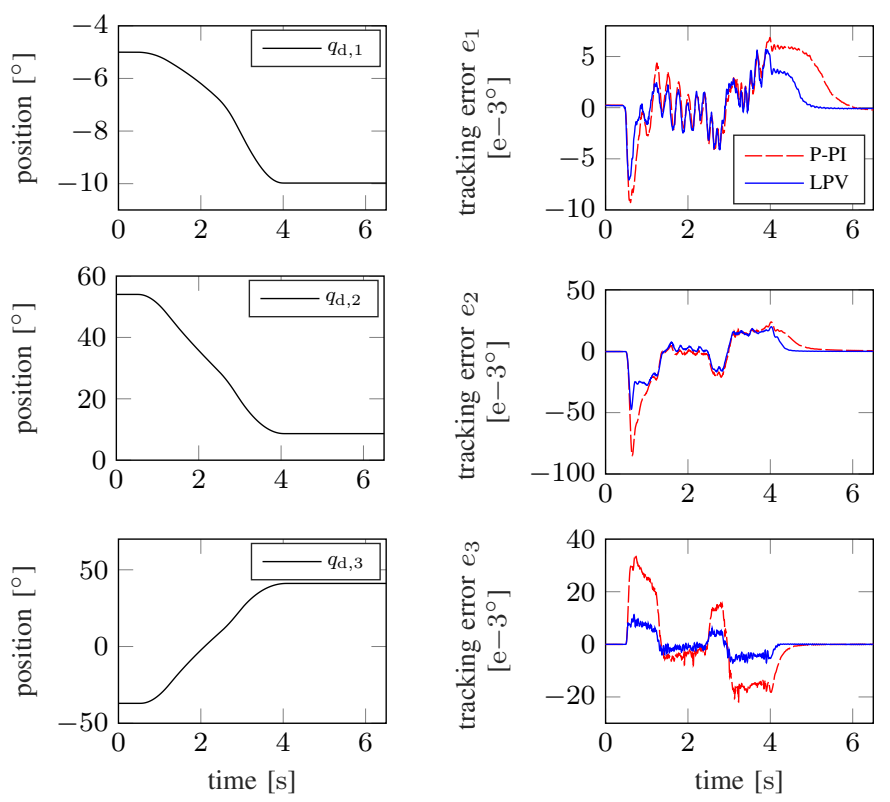

Fig. 10. Experimental Cartesian trajectory tracking performance of the P-PI and LPV control in the time domain.

TABLE IV

EXPERIMENTAL CONTROL PERFORMANCE

\begin{tabular}{ccccccc}
\hline \multirow{2}{*}{ Joint $i$} & $\left\|e_{i}\right\|_{1}\left[\mathrm{e}-3^{\circ}\right]$ & $\Delta_{1}[\%]$ & \multicolumn{2}{c}{$\left\|e_{i}\right\|_{\infty}\left[\mathrm{e}-3^{\circ}\right]$} & $\Delta_{\infty}[\%]$ \\
\cline { 2 - 3 } \cline { 5 - 6 } & $\mathbf{P}-\mathbf{P I}$ & $\mathbf{L P V}$ & & $\mathbf{P - P I}$ & $\mathbf{L P V}$ & \\
\hline 1 & 16.6 & 9.6 & -42 & 9.3 & 7.1 & -24 \\
2 & 69.2 & 48.3 & -30 & 85.6 & 47.8 & -54 \\
3 & 49.3 & 16.2 & -67 & 46.3 & 12.7 & -72 \\
\hline
\end{tabular}

$[x, y, z]=[2700,-235,975] \mathrm{mm}$ (cf. Fig. 8a), intermediate $2100,-235,975] \mathrm{mm}$ (cf. Fig. 8b) and the final position $[1500,-235,975] \mathrm{mm}$ (cf. Fig. 8c) given in the robot base frame. The corresponding desired joint positions $q_{\mathrm{d}, i}$ and tracking errors $e_{i}$ of the P-PI [26] and the proposed LPV controller are shown in Fig. 10. The variation of the manipulator configuration alters the system behavior during the trajectory $\left(-0.72 \leq \rho_{1} \leq 0.23,-0.84 \leq \rho_{2} \leq 0.91\right)$. As expected, the proposed LPV controller outperforms the P-PI controller regarding the $\mathcal{L}_{\infty}$ as well as the $\mathcal{L}_{1}$ norm (cf. Table IV). 


\section{CONCLUSION}

This paper presented a gain-scheduled control approach for industrial robots based on a LPV model. Both, a LPV model and a related system identification method were proposed. Based on the identified model, an adaptive controller was designed, which allows systematic stability testing while maintaining a simple control structure. The experimental results on an industrial robot with heavy payload demonstrate the effectiveness of the control approach despite the model uncertainty. Therefore, the LPV technique seems promising for the control of IRs in industrial settings.

Current research concerns the application and evaluation of the control approach during machining.

\section{REFERENCES}

[1] L. Sweet and M. Good, "Re-definition of the robot motion control problem: Effects of plant dynamics, drive system constraints, and user requirements," in The 23rd IEEE Conference on Decision and Control, pp. 724-732. IEEE, 1984.

[2] I. Iglesias, M. A. Sebastián, and J. E. Ares, "Overview of the state of robotic machining: Current situation and future potential," Procedia Engineering, vol. 132, pp. 911-917, 2015.

[3] A. Verl, A. Valente, S. Melkote, C. Brecher, E. Ozturk, and L. T. Tunc, "Robots in machining," CIRP Annals, vol. 68, no. 2, pp. 799-822, 2019.

[4] R. Bogue, "Cutting robots: a review of technologies and applications," Industrial Robot: An International Journal, vol. 35, no. 5, pp. 390-396, 2008.

[5] P. M. Bhatt, R. K. Malhan, A. V. Shembekar, Y. J. Yoon, and S. K. Gupta, "Expanding capabilities of additive manufacturing through use of robotics technologies: A survey," Additive Manufacturing, vol. 31, p. 100933,2020

[6] M. W. Spong, "Modeling and control of elastic joint robots," Journal of Dynamic Systems, Measurement, and Control, vol. 109, no. 4, pp. 310-318, 1987.

[7] P. Tomei, "A simple pd controller for robots with elastic joints," IEEE Transactions on Automatic Control, vol. 36, no. 10, pp. 1208-1213, 1991.

[8] B. Brogliato, R. Ortega, and R. Lozano, "Global tracking controllers for flexible-joint manipulators: a comparative study," Automatica, vol. 31, no. 7, pp. 941-956, 1995

[9] A. de Luca, "Feedforward/feedback laws for the control of flexible robots," in Robotics and Automation, 2000 IEEE International Conference, pp. 233-240. Piscataway: I E E E, 2000.

[10] B. Siciliano and O. Khatib, Springer Handbook of Robotics. Cham: Springer International Publishing, 2016.

[11] J. Kim and E. A. Croft, "Preshaping input trajectories of industrial robots for vibration suppression," Robotics and Computer-Integrated Manufacturing, vol. 54, pp. 35-44, 2018.

[12] L. Dai, Y. Yu, D.-H. Zhai, T. Huang, and Y. Xia, "Robust model predictive tracking control for robot manipulators with disturbances," IEEE Transactions on Industrial Electronics, vol. 68, no. 5, pp. 42884297, 2021.

[13] T. A. Khaled, O. Akhrif, and I. A. Bonev, "Dynamic path correction of an industrial robot using a distance sensor and an adrc controller," IEEE/ASME Transactions on Mechatronics, vol. 26, no. 3, pp. 16461656, 2021.

[14] K. Shojaei, A. Kazemy, and A. Chatraei, "An observer-based neural adaptive \$pid^2\$ controller for robot manipulators including motor dynamics with a prescribed performance," IEEE/ASME Transactions on Mechatronics, vol. 26, no. 3, pp. 1689-1699, 2021.

[15] J. Hu, C. Li, Z. Chen, and B. Yao, "Precision motion control of a 6-dofs industrial robot with accurate payload estimation," IEEE/ASME Transactions on Mechatronics, vol. 25, no. 4, pp. 1821-1829, 2020.

[16] Y. Pan, H. Wang, X. Li, and H. Yu, "Adaptive command-filtered backstepping control of robot arms with compliant actuators," IEEE Transactions on Control Systems Technology, vol. 26, no. 3, pp. 1149$1156,2018$.

[17] Y. Liu, Z. Li, H. Su, and C.-Y. Su, "Whole-body control of an autonomous mobile manipulator using series elastic actuators," IEEE/ASME Transactions on Mechatronics, vol. 26, no. 2, pp. 657-667, 2021.
[18] J. Kim and E. A. Croft, "Full-state tracking control for flexible joint robots with singular perturbation techniques," IEEE Transactions on Control Systems Technology, vol. 27, no. 1, pp. 63-73, 2019.

[19] A. Albu-Schäffer, C. Ott, and G. Hirzinger, "A unified passivity-based control framework for position, torque and impedance control of flexible joint robots," The International Journal of Robotics Research, vol. 26, no. 1, pp. 23-39, 2007.

[20] F. Petit and A. Albu-Schaffer, "State feedback damping control for a multi dof variable stiffness robot arm," in 2011 IEEE International Conference on Robotics and Automation, pp. 5561-5567. IEEE, 2011.

[21] M. Iskandar, C. Ott, O. Eiberger, M. Keppler, A. Albu-Schaffer, and A. Dietrich, "Joint-level control of the dlr lightweight robot sara," in 2020 IEEE/RSJ International Conference, pp. 8903-8910. IEEE, 2020.

[22] J. W. Sensinger and J. H. Lipsey, "Cycloid vs. harmonic drives for use in high ratio, single stage robotic transmissions," in IEEE International Conference on Robotics and Automation (ICRA), 2012, pp. 4130-4135. Piscataway, NJ: IEEE, 2012.

[23] P. Mesmer, M. Neubauer, A. Lechler, and A. Verl, "Challenges of linearization-based control of industrial robots with cycloidal drives," in 2021 IEEE International Conference on Mechatronics (ICM), pp. 18. IEEE, 2021.

[24] T. Brogårdh, "Robot control overview: An industrial perspective," Modeling, Identification and Control: A Norwegian Research Bulletin, vol. 30, no. 3, pp. 167-180, 2009.

[25] Tsai et al., Secondary Position feedback control of a robot. United States Patent: US20100191374A1, 2013.

[26] P. Mesmer, M. Neubauer, A. Lechler, and A. Verl, "Robust design of independent joint control of industrial robots with secondary encoders," Robotics and Computer-Integrated Manufacturing, vol. 73, p. 102232, 2022.

[27] W. J. Rugh and J. S. Shamma, "Research on gain scheduling," Automatica, vol. 36, no. 10, pp. 1401-1425, 2000.

[28] J. S. Shamma and M. Athans, "Analysis of gain scheduled control for nonlinear plants," IEEE Transactions on Automatic Control, vol. 35, no. 8, pp. 898-907, 1990.

[29] C. Hoffmann and H. Werner, "A survey of linear parameter-varying control applications validated by experiments or high-fidelity simulations," IEEE Transactions on Control Systems Technology, vol. 23, no. 2, pp. 416-433, 2015.

[30] A. Forrai, T. Ueda, and T. Yumura, "Electromagnetic actuator control: A linear parameter-varying (lpv) approach," IEEE Transactions on Industrial Electronics, vol. 54, no. 3, pp. 1430-1441, 2007.

[31] D. Sepasi, R. Nagamune, and F. Sassani, "Tracking control of flexible ball screw drives with runout effect and mass variation," IEEE Transactions on Industrial Electronics, vol. 59, no. 2, pp. 1248-1256, 2012.

[32] P. Mesmer, M. Neubauer, A. Lechler, and A. Verl, "Drive-based vibration damping control for robot machining," IEEE Robotics and Automation Letters, vol. 5, no. 2, pp. 564-571, 2020.

[33] P. Apkarian, P. Gahinet, and G. Becker, "Self-scheduled h $\infty$ control of linear parameter-varying systems: a design example," Automatica, vol. 31, no. 9, pp. 1251-1261, 1995.

[34] M. Gautier and W. Khalil, "Direct calculation of minimum set of inertial parameters of serial robots," IEEE Transactions on Robotics and Automation, vol. 6, no. 3, pp. 368-373, 1990.

[35] Jan Swevers, Walter Verdonck, and Joris De Schutter, "Dynamic model identification for industrial robots," IEEE Control Systems, vol. 27, no. 5, pp. 58-71, 2007.

[36] J. Doyle, B. A. Francis, and A. R. Tannenbaum, "Feedback control theory," in Servo motors and industrial control theory, ser. Mechanical Engineering Series, R. Firoozian, Ed., pp. 1-16. New York: Springer, 2009.

[37] H. K. Khalil, Nonlinear systems, 3rd ed. Upper Saddle River, NJ: Prentice Hall, 2002.

[38] G. Becker and A. Packard, "Robust performance of linear parametrically varying systems using parametrically-dependent linear feedback," Systems \& Control Letters, vol. 23, no. 3, pp. 205-215, 1994.

[39] P. Gahinet, P. Apkarian, and M. Chilali, "Affine parameter-dependent lyapunov functions and real parametric uncertainty," IEEE Transactions on Automatic Control, vol. 41, no. 3, pp. 436-442, 1996.

[40] MathWorks, Robust Control Toolbox: User's Guide. The MathWorks, Inc., Natick, MA, 2020.

[41] S. Skogestad and I. Postlethwaite, Multivariable feedback control: Analysis and design, 2nd ed. Chichester: Wiley, 2009.

[42] P. Apkarian, M. N. Dao, and D. Noll, "Parametric robust structured control design," IEEE Transactions on Automatic Control, vol. 60, no. 7, pp. 1857-1869, 2015. 


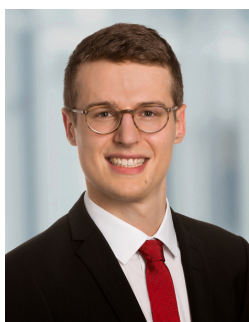

Patrick Mesmer was born in Tettnang, Germany, in 1993. He received the B.Eng. degree in electrical engineering from the Baden-Württemberg Cooperative State University, Ravensburg, Germany, and the M.Sc. degree in electrical engineering from University of Stuttgart, Stuttgart, Germany, in 2015 and 2018, respectively, where he is currently pursuing the Ph.D. (Dr-Ing.) degree under the supervision of A. Verl.

His current research interests include modeling, ing on machining applications. control of industrial robots focus

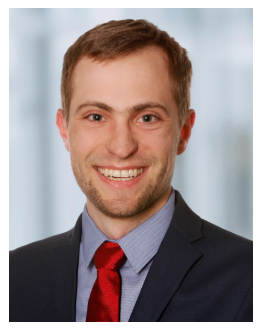

Christoph Hinze was born in Filderstadt, Germany, in 1991. He revecived his M.Sc. degree in engineering cybernetics with a main focus in system dynamics from the University of Stuttgart, Germany, where he is currently pursuing the Ph.D. (Dr-Ing.) degree under the supervision of A. Verl.

His current research interests include control methods for dynamics enhancement of drive systems and model-based identification.

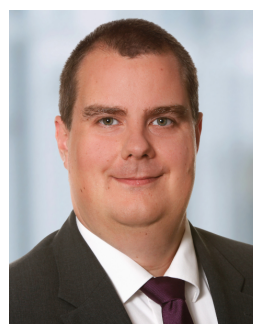

Armin Lechler was born in Bietigheim-Bissingen, Germany, in 1979. He received the Dipl.-Ing. degree in engineering cybernetics with emphasis on production engineering from the University of Stuttgart, Stuttgart, Germany, in 2006.

He was a Research Assistant at the Institute for Control Engineering of Machine Tools and Manufacturing Units (ISW), University of Stuttgart, in 2009 , where he was involved in research in information and communication technology. From 2009 to 2011 , he was the Head of the Department Control Engineering, ISW. Since 2009, he is the Managing Director of FISW Steuerungstechnik GmbH, Stuttgart. In 2011, he joined ISW as the Managing Chief Engineer and received the Dr.-Ing. degree. Since 2013, he has been the Deputy Director of ISW.

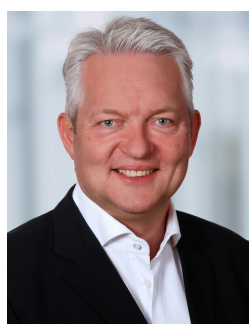

Alexander Verl was born in Frunse, Russia, in 1966. He received the Dipl.-Ing. degree in electrical engineering from Friedrich-Alexander-University Erlangen-Nürnberg, Erlangen, Germany, in 1991, and the Dr.-Ing. degree in control engineering from the DLR Institute of Robotics and Mechatronics, Oberpfaffenhofen, Germany, in 1997.

From 1997 to 2005, he was Founder and Managing Director of AMATEC Robotics GmbH (part of KUKA Roboter GmbH since 2005), Augsburg, Germany. Since 2005, he has been a Full Professor with the University of Stuttgart, Stuttgart, Germany, where he was the Head of the Institute for Control Engineering of Machine Tools and Manufacturing Units.

Prof. Verl received the Diesel Gold Medal from the German Institute of Inventions in 2014, the Julius von Haast Fellowship Award from the Royal Society of New Zealand in 2012, the Honorary Doctoral degree (Dr.h.c.) of the Technical University of Cluj-Napoca, Romania, in 2012, the Honorary Professor of The University of Auckland, New Zealand, in 2012, the Invention and Entrepreneurship Award of the IEEE Robotics and Automation Society, the International Federation of Robotics in 2010, and the Dr.h.c. of the Politehnica University of Timisoara, Romania, in 2009. 


\section{LIST OF FIGURES}

1 KUKA KR210-2 IR testbench with SEs. . . . .

2 Measured and identified motor torque of the first three joints of the KUKA KR210 of the rigid body dynamics identification. . . . . . . . . . Identified diagonal mass matrix elements $\left(M_{33} \approx 243.9 \mathrm{kgm}^{2}\right) . \ldots \ldots \ldots \ldots$ Comparison of the magnitude frequency response of the first three joints of the KUKA KR210-2 (blue) and the identified joint model (red) (input: $u_{i}{ }^{-1} \dot{\theta}_{i}$, output: $\left.\dot{q}_{i}\right) \ldots \ldots \ldots \ldots$ Structure for the LPV controller design (based on [33]). . . . . . . . . . . . . . . Block diagram of the LPV plant including the drive-based damping control. . . . . . . . . . . . Block diagram of control architecture for the experimental validation. . . . . . . . . .

8 Manipulator configurations for experimental val-

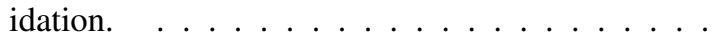

9 Sensitivity (left) and complementary sensitivity function (right) of position control loop of the P-PI (dashed) and LPV (solid) controller of the first joint for different manipulator configurations (red: Fig. 8a, blue: Fig. 8b, black: Fig. 8c). . . .

10 Experimental Cartesian trajectory tracking performance of the P-PI and LPV control in the time domain.

\section{LIST OF TABLES}

4

6

I Identified elastic parameters of the KUKA KR210-2 ................ 4

II LPV controller gains for the KUKA KR210-2 . 6

7 III Mass matrix entries of the KUKA KR210-2 of specified manipulator configuration (cf. Fig. 8) . 7

7 IV Experimental control performance . . . . . . 7 